\title{
Hands-Free Control Interfaces for an Extra Vehicular Jetpack
}

\author{
Jennifer Rochlis Zumbado \\ NASA Johnson Space Center \\ 2101 NASA Parkway \\ Houston, TX 77058 \\ 281-483-1718 \\ jennifer.l.rochlis@nasa.gov
}

\author{
Pedro H. Curiel \\ NASA Johnson Space Center \\ 2101 NASA Parkway \\ Houston, TX 77058 \\ 281-244-7209 \\ pedro.h.curiel@nasa.gov
}

\author{
Sam Schreiner \\ University of Minnesota \\ Minneapolis, MN 55414 \\ 651-485-4539 \\ schr0910@umn.edu
}

\begin{abstract}
The National Aeronautics and Space Administration (NASA) strategic vision includes, as part of its long-term goals, the exploration of deep space and Near Earth Asteroids (NEA). To support these endeavors, funds have been invested in research to develop advanced exploration capabilities. To enable the human mobility necessary to effectively explore NEA and deep space, a new extravehicular activity (EVA) Jetpack is under development at the Johnson Space Center. The new design leverages knowledge and experience gained from the current astronaut rescue device, the Simplified Aid for EVA Rescue (SAFER). Whereas the primary goal for a rescue device is to return the crew to a safe haven, in-space exploration and navigation requires an expanded set of capabilities. To accommodate the range of tasks astronauts may be expected to perform while utilizing the Jetpack, it was desired to offer a hands-free method of control. This paper describes the development and innovations involved in creating two hands-free control interfaces and an experimental test platform for a suited astronaut flying the Jetpack during an EVA.
\end{abstract}

\section{TABLe OF Contents}

1. INTRODUCTION .1

2. HANDS-FreE METHOd SELECTION .................2 3. SoftWARE OVERVIEW AND DESIGN................4 5. Controller Hardware .................................6 6. TEST BED HARDWARE........................................6

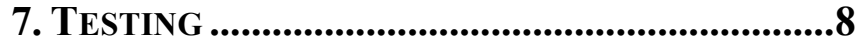
8. Conclusions ANd Future Testing..............9 REFERENCES.................................................99 BIOGRAPHIES.........................................................10

\section{INTRODUCTION}

As NASA plans to visit Near Earth Asteroids (NEA) and outer moons as part of their strategic vision, new capabilities for human exploration and mobility are required. Specifically, as these objects do not have the surface gravity necessary to allow humans to land on and explore the surface on foot, an Extravehicular Activity (EVA) mobility solution is needed. NASA flew the first astronaut propulsive system, the Manned Maneuvering Unit (MMU), on the Space Shuttle in 1984 on STS-41B, STS$41 \mathrm{C}$ and STS-51A. It was retired from use after these missions, and astronauts remained tethered or were carried by the Space Shuttle robotic arm until its successor, the Simplified Aid for EVA Rescue (SAFER) was flown in
1994 [1]. However, as the SAFER unit was intended to act as an emergency rescue device only, it was not designed to provide primary EVA mobility. The approach for this current Jetpack effort was to leverage the existing SAFER avionics system, redesign the mechanical and propulsive systems, and add functionality in the form of a new handsfree method of control (replacing the existing hand controller). This hands-free control method will enable astronauts to command their motion while transporting payloads and conducting two-handed tasks. While any flight design will eventually have to be integrated with an EVA suit, the goal for this initial activity was to think "outside the box" in terms of current capabilities and limitations of existing and developmental space suits.

As described in further detail in section 2, literature review and preliminary research led to the selection of voice and foot sensor controls for the input devices. The voice command modality utilizes a wearable headset with wireless communications, an adjustable operator display screen, microphone and headset. The foot sensor design is derived from deep-sea diving applications of foot pedal controls, adapted for inclusion into an astronaut's space suit boot worn during a microgravity EVA. The overall objectives were to provide proof of concept by conducting an engineering evaluation of the system using a new 1-g analog Air Bearing Floor (ABF) test facility [2]. This paper will describe the design process for the Jetpack, focusing on the hand-free control methods, air bearing test sled assembly, initial engineering evaluation and future work.

Before researching and selecting the hands-free control modalities, the team determined what functionality the existing avionics afforded and which hand controller capabilities needed to be retained in the new implementation. An early critical milestone was to establish communication with the legacy avionics unit and decipher its communication protocol in order to mimic hand controller commands. Similarly, an examination of the existing SAFER ABF test facility performance drove the requirements for our next generation unit. The SAFER $\mathrm{ABF}$ air sled revealed that although the amount of motion the thrusters provide is sufficient for in-space propulsion, the dynamics and friction that accompany air bearing testing in 1-g are difficult to overcome with the limited propulsive force provided by the thrusters. To increase the quality and efficiency of the demonstration and testing, a new ABF sled was designed and built (as well as a parallel effort to

U.S. Government work not protected by U.S. copyright 
redesign the thruster system as part of the Jetpack effort). Figure 1 shows a computer model of the new jetpack concept. The suit is a rear-entry concept suit with an interface plate (SIP) that mates to the spacecraft, and an integrated Portable life support system (PLSS, upper gold box). The thruster assembly is housed in the lower green and grey section behind the astronaut waist and hips. Upper thruster pods can be seen on either side of the helmet, mounted to the SIP, and the remaining thrusters are located on the jetpack lower housing. To the right in Figure 1 is the legacy SAFER hardware test sled. The test being performed in the photo is the initial voice command checkout with a commercial noise-cancelling microphone. Protocols and software were verified using this original system before the new jetpack test sled was completed.

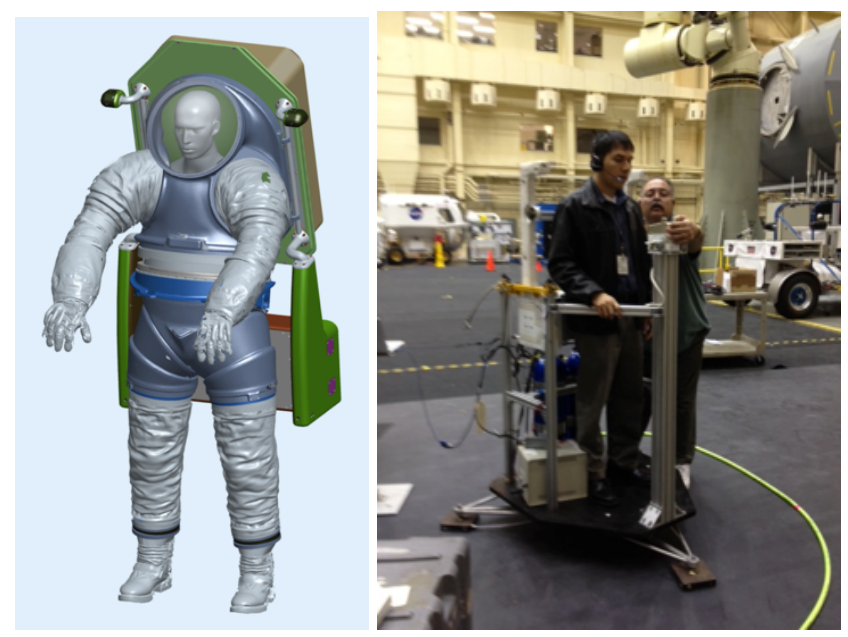

Figure 1 - Left: A computer model of the new jetpack design. Right: Early voice control testing with the existing SAFER test sled

\section{HANDS-Free Method Selection}

In order to develop a hands-free control method, it is important to understand how the existing flight units are commanded, how astronauts are trained to use the system, and the functionality required of a mobility device.

\section{Hand Controller Module}

The SAFER and MMU are piloted using a Hand Controller Module (HCM). This module requires two hands to hold and stabilize the unit during operations and houses both a joystick for thruster commands and a liquid crystal display (LCD) for monitoring system status received from the avionics. The four-axis joystick can be used in either translation or rotation mode; a physical switch must be thrown to change the control mode. The six degrees of freedom available in a microgravity environment are mapped via a body-centric coordinate frame. Axes are mapped with astronaut forward/backward as +/-X (Roll about $\mathrm{X}$ ), right/left as $+/$-Y (Pitch about Y) and up/down as $1+Z$ (Yaw about $Z$ ). In either translation or rotation mode, there is a central null position and a fourth corollary axis always available. Figure 2 shows the HCM labeled with translation mode axes. Note the availability of the pitch axis in translation mode; the $+/-\mathrm{X}$ axis is likewise available in rotation mode [3].

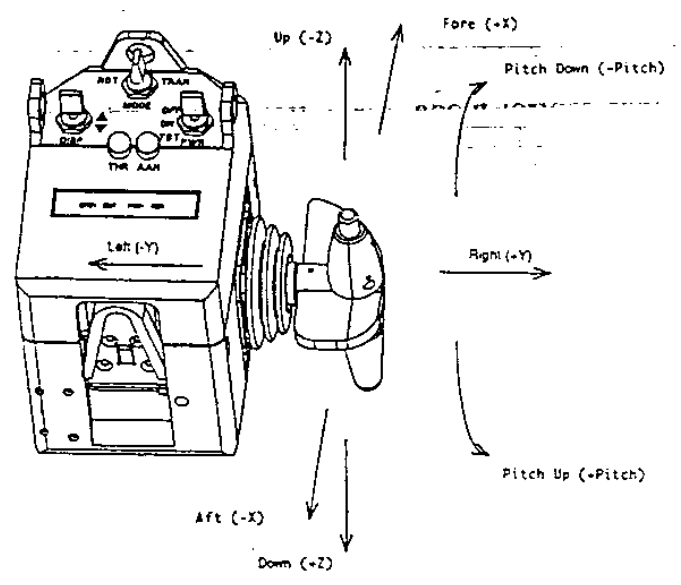

\section{Figure 2 SAFER Hand Controller Module showing the command axes in translation mode}

Additional switches and indicators include a thruster cue light (lit when a "thruster-on" condition is detected by the avionics software), an indicator for when Automatic Attitude Hold (AAH) is enabled for one or more rotation axes, a power/test switch and a display switch to scroll through information on the LCD. The SAFER and new Jetpack house 24 thrusters (four for each degree of freedom). As long as the joystick is depressed away from the null position in any axis, a command is sent to the avionics that fires the appropriate four thrusters. It is possible therefore, to hold the joystick in a given axis and increase speed until the cold gas is depleted from the jetpack tanks. Astronauts are taught specific operational techniques to increase safety, maximize consumables and maintain the highest level of command authority and situation awareness (SA). A discrete short pulse method for flying SAFER is taught, as any motion input to the system must be countered before the system can come to a stop. To minimize the number of DOF that the astronaut has to control, the $\mathrm{AAH}$ can be enabled and will hold rotation (based on Inertial Measurement Unit data) while maintaining translation authority. Lastly, the hand controller has a small two-line LCD that shows basic status and telemetry data such as the tank pressure, and battery voltage.

\section{Human as an Output Device}

Hands-free control is intended for use when an astronaut is engaged in two-handed tasks (such as tool handling, photography, or carrying larger payloads). An interface must be provided that preserves the relevant functionality previously provided by the HCM, but it can also increase the operator experience and usability by taking advantage of advanced techniques. The hands-free control methods selected were designed to enhance operator Situation Awareness (SA) and support the desired discrete pulse method. Interviews with multiple SAFER trainers and 
astronauts led us to an appreciation of the necessary functionality, limitations, the "nice to haves" and astronaut preferences based on their flight experience.

Without the use of hands, alternate modalities must be exploited for commanding. Several biomedical mechanisms (utilized extensively within the accessible and wearable computing communities) allow humans to act as output devices [4], [5], [6], [7], [8]. Electro-physical signal options include muscle activation (electro-chemical, electromyography and/or kinetic), brain wave activation, galvanic skin resistance and biofeedback. Additional options include audio output, speech and non-speech, and gesture recognition (eye tracking, electrooculography, face, lip, nose and limb). These modalities vary in terms of ease of use, reliability, data processing requirements, maturity, robustness and compatibility with the jetpack and spacesuit.

When trading these modalities for an in-space application, several factors must be considered. First, spacesuit kinematics limit joint motion and gesture performance. Recognition systems typically require external and fixed cameras or viewing systems that are not operationally applicable in a free-flight situation. Current gesture systems are emerging and immature, resulting in lower levels of accuracy and reliability. Gestures can be fatiguing to perform over prolonged periods of time, and are particularly compounded when working against the dynamics of a pressurized spacesuit. Biofeedback methods require significant training and large amounts of signal processing. For a real-time application, this would introduce signal noise and reduce signal availability. Speech control must be designed to consider environmental noise constraints. In general, any hands-free method that must be integrated into the environment of the spacesuit and jetpack hardware should be wearable, multi-modal (to accommodate multiple control functions), and require no mechanical linkages between the operator and the input device.

\section{Voice Control}

We chose to proceed to the initial design phase with two distinct control concepts. The first was to utilize natural language speech commands as inputs to the SAFER control avionics. A verbal command taxonomy was developed to control the 6 DOF available to the astronaut. It leveraged the language used to teach SAFER operations to astronauts during their flight training, that of "plus X" or "minus yaw" for example. Additional widely used single-word taxonomies were also considered, such as the familiar bodycentric directions "up, down, left, right, forward, backward". This is sufficient for translational motions, but as humans do not have a similar standard daily-life vocabulary for rotational directions, each rotation command would also have to be accompanied by a direction command (for example, spin right, or pitch down). This could lead to redundancies in the command streams and recognition errors (i.e. mistaking "spin right" for "right"). A benefit of voice commanding is that it mimics the current paradigm of discrete input control that both astronauts and trainers felt was vital to maintain for safety and efficiency.

While voice control alone would be sufficient to simply fire the jetpack thrusters, the HCM display parameters also needed accommodation. Research into available Commercial-off-the-shelf (COTS) solutions for rapid prototyping led to a hardware device housing microphone, headphone, and display capabilities. The "Golden-i®" headset, shown in Figure 3, is a development unit created I partnership between Motorola and Kopin. This unit was chosen for several attributes; it's wearable, adjustable, wireless and can easily be integrated into a spacesuit without modification. The headset is a lightweight (six ounces), Bluetooth/WiFi head mounted computer with a 15 inch virtual PC display that can be worn below the left or right eye. A near-ear speaker and microphone enable voice control (using VoCon 3200 speech recognition software) of the windows operating computer system and display. A 6axis solid-state real-time position tracker is available and can be used to control cursor and screen positioning, for example [9]. One final reason for selection this unit was its extensibility to incorporate future concepts such as advanced graphics and egocentric situation awareness aids. Section 3 will describe further details on the software and integration of the unit.

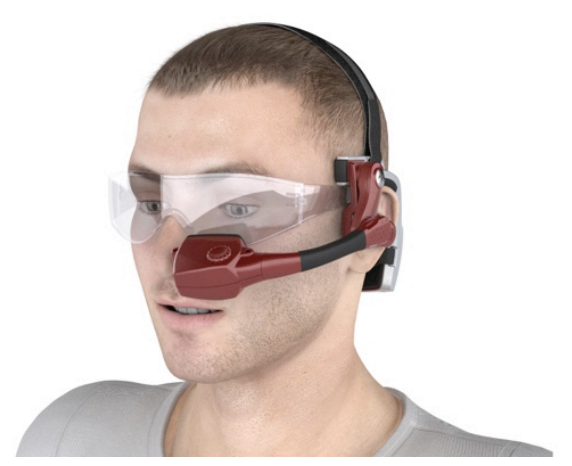

Figure 3 - Golden-i® developer unit headset

\section{Foot Control}

The second concept chosen for initial development was foot sensor control. This is based on methods used in Deep Worker Sub and Atmospheric Diving Suit applications (see Figure 4). Competency is reportedly achieved for 2-pedal control of 2 or 3 DOF within approximately 20 hours of training (and many more to reach proficiency) [10]. The EVA suit does not provide a surface on which to reach forces, nor does it allow for significant ankle flexion within the boot - both necessary for a pedal device. Therefore the concept was adapted for pedal-free, friction-free, traininglight commanding. Additional requirements are to 1) provide discrete on/off thruster control for the three axes of motion available during an air-bearing test $(+/-\mathrm{X},+/-\mathrm{Y}$ and +/-Yaw), 2) create a system suitable for various 
anthropometries and 3) provide a robust system for demonstration purposes.
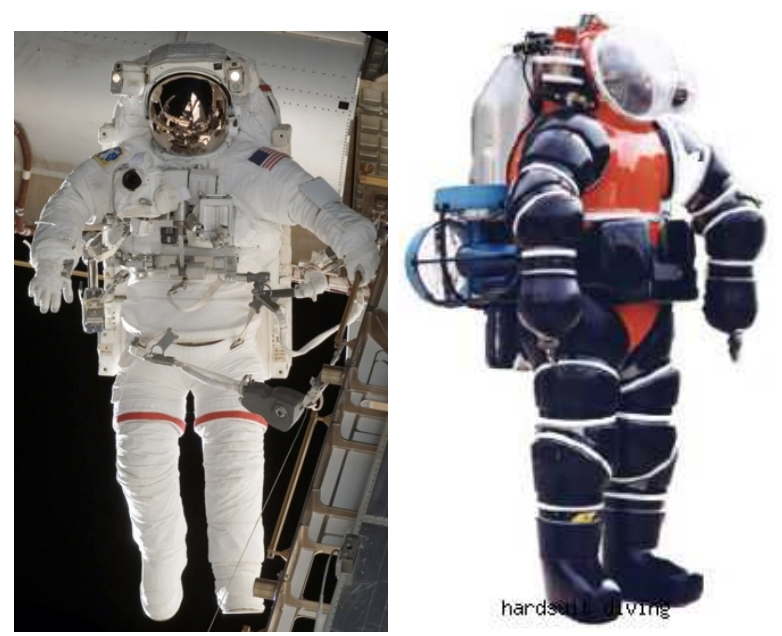

Figure 4 - Left: NASA EVA suit and SAFER system. Right: OceanWorks Hard Diving Suit

Beginning with the constraints of the EVA suit, research led to the use of individual for sensors rather than "shoe insert" type pressure mapping systems. Full inserts provide far more data than is necessary for discrete control, requires significant data filtering and processing and will compromise the real-time command requirement. Tekscan FlexiForce 1-inch diameter sensors with a response time less than 5 microseconds and a 0-25 $\mathrm{lb}$ force range were selected for proof-of-concept. Six sensors total (three per foot) supplies the mapping (see Figure 5) to the DOF mentioned previously. Pressing down on the big toe of the right foot commands $+X$, pressing on the right ball of the foot commands $-\mathrm{X}$, similarly for $+/-\mathrm{Y}$ on the left foot, and the outer edges of the right and left foot command $+/$-Yaw respectively. Although ultimately integrated into a spacesuit boot, demonstration and engineering evaluation of the sensors is done in a shirtsleeve environment (unsuited). As such, the sensors must initially be designed for placement on the test sled rather than within a spacesuit boot. Future work described in section 8 will outline some future plans to integrate the sensors into a spacesuit boo. The test sled mounting strategy will be described further in section 6 .
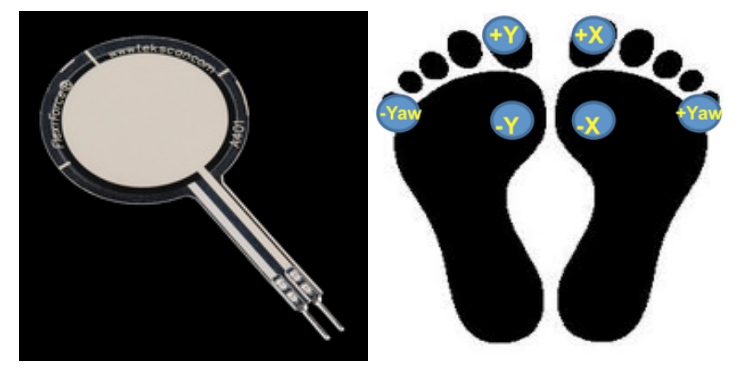

Figure 5 - Left: flexi force sensor and Right: positioning of the sensors under the foot labeled with the directional mapping

\section{SOFTWARE OVERVIEW AND DESIGN}

This section will outline the software engineering that enabled the hands-free controller to function with the SAFER avionics system and command the thrusters, as well as the development of the headset code. The legacy SAFER software was written in Borland $\mathrm{C}$ and designed to run on Windows 95, which resulted in an incompatibility with modern test hardware and software. With the aid of the source code and the legacy SAFER documentation, the communication protocol used to interface with the SAFER avionics unit was translated to a more recent language that newer hardware could execute. $\mathrm{C \#}$ was selected as the programming language because it is based on the .NET framework, which is advantageous in that its communication classes are easier to implement and it is not hardware specific, so it can be seamlessly transferred to other computers for operation.

The functional block diagram for the software setup is displayed in Figure 6, illustrating the relationship between the various software and hardware components. The updated software is loaded onto a server laptop running Windows 7 that interfaces with the hands-free controller hardware. The server laptop manages three different data streams to integrate the two hands-free input methods with the existing avionics code and connect to the jetpack. First, it controls a one-way data stream to the foot sensors, through which it receives thruster fire commands. Second, it manages a two-way data stream to the headset, through which it receives thruster commands while also sending telemetry data to the visual display on the headset. The third data stream exists between the server laptop and avionics box. Through this data stream, the server laptop issues thruster commands to the avionics box while also periodically requesting and receiving telemetry data. The telemetry data includes the status of different parameters in the system including mode (translation or rotation), AAH status, battery capacity, propellant capacity, etc.

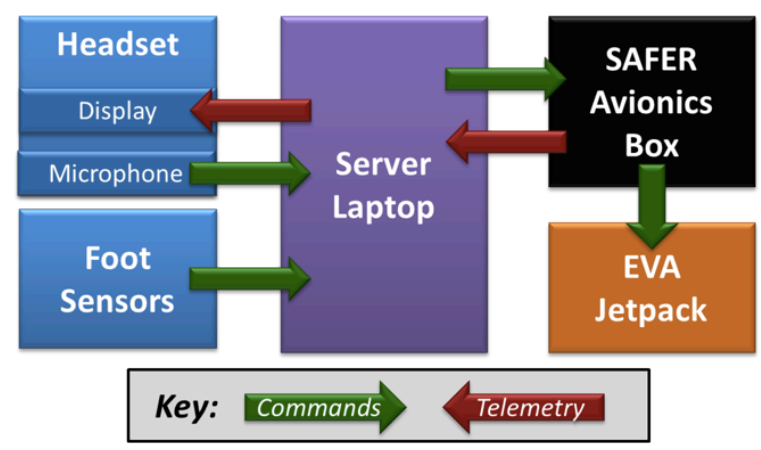

Figure 6 - Functional Block Diagram of the jetpack hardware and software

The functionality of the software on the server laptop is implemented in a Graphical User Interface (GUI), shown in Figure 7. Though the server laptop can facilitate the interaction between the jetpack avionics box and control inputs without any graphical display, the GUI was 
developed to provide situation awareness, system diagnostics and aid in troubleshooting. The content of the GUI on the server laptop (herein referred to as the "server GUI") is derived from a combination of existing avionics code used in previous jetpack testing and open-source Wi-Fi communication code. The GUI has separate display panels for the telemetry data, wireless network status, thruster command sending status, and thruster fire confirmation. Additionally, there are panels that monitor the connection to both hands-free control inputs and a command log that records all activity managed by the server laptop.

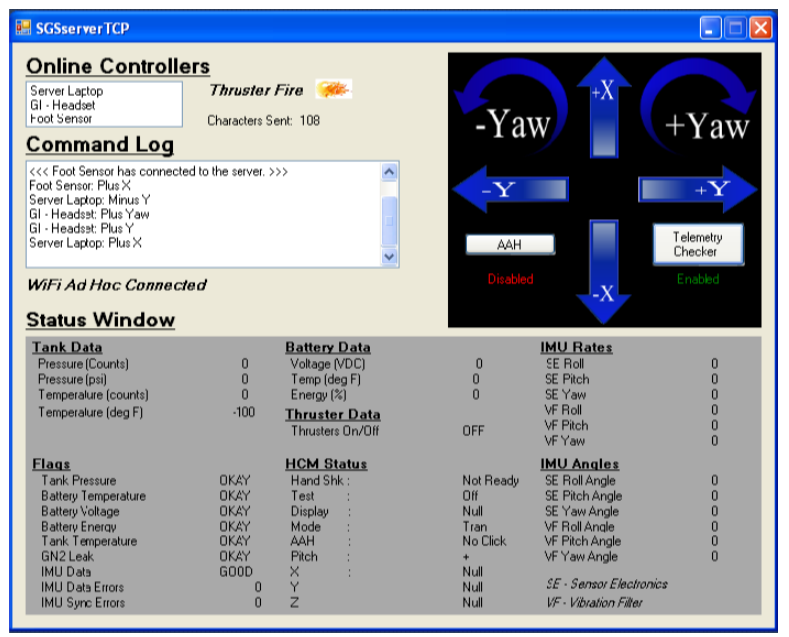

Figure 7 - Jetpack engineering GUI

The server GUI has a dual-functionality in that in addition to relaying thruster commands from the hands-free controllers, it can also issue thruster commands directly to the avionics box without any outside controller input. This is not intended to be a primary means for controlling the jetpack, but rather an avenue for troubleshooting should a problem arise with either of the control inputs. Behind the scenes, the server GUI routinely checks the status of the thruster supply tanks, battery, and other important quantities in the telemetry data. This process involves requesting telemetry data from the avionics box, receiving it, processing it, displaying it in the server GUI, and relaying it onto to the headset display for the operator to observe. A button on the server GUI allows the user to turn the periodic telemetry check on or off. Furthermore, the physical switch on the HCM that sets and releases the AAH was converted into a virtual button on the GUI. In this way, during testing and checkout, the server can change the AAH setting via the button or the headset operator can change the AAH setting with a voice command.

The server laptop's GUI also had to be replicated for the headset unit. This was not a trivial task, in that the code was not as portable as was anticipated. Although both the headset and server laptop used the same .NET framework, the .NET micro framework on the headset is only a subset of the full framework on the laptop. Some functions used in the laptop software therefore, were not available for the headset and workarounds had to be developed. The headset uses its own customized software running on the Windows mobile platform. Figure 8 shows the appended GUI that is displayed to the operator in the headset during voice commanding (this could also be work during foot commanding as well). It gives feedback on the receipt of the voice command (also provided verbally through the headset speaker) as well as a positive indication of thruster fire. It also shows a limited set of status information, and a thruster input count, such that the astronaut is aware of how much command authority has been input (and therefore needs to be "backed-out").

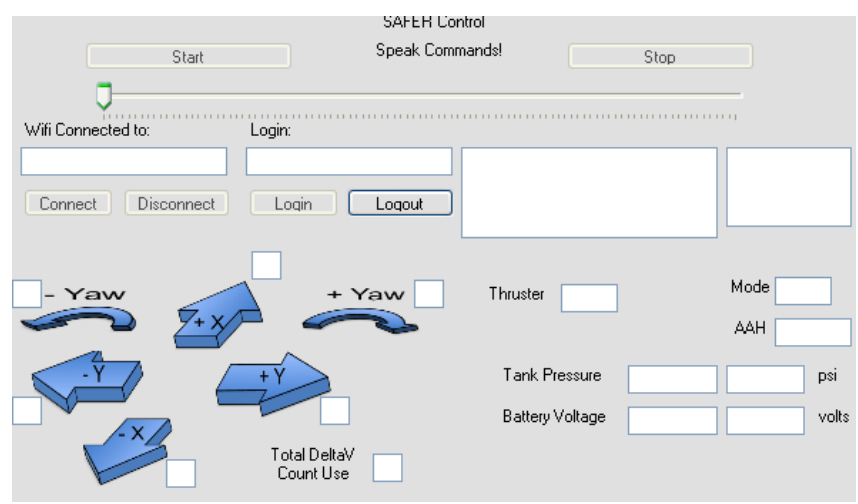

Figure 8 - Operator GUI displayed in the headset

\section{Software Design Considerations}

While developing the software to run on the server laptop, there were a number of components implemented into the code to ensure the jetpack functioned in a responsive and safe manner during testing. The original legacy software application could either send commands one at a time or read telemetry, but not both simultaneously. Considerable time was spent ensuring that the server GUI could manage its multiple responsibilities without losing responsiveness. For instance, consider a scenario in which the server laptop is busy requesting, receiving, processing, and relaying telemetry data when a thruster command is issued from an input device, perhaps to avoid a dangerous situation where immediate action is required. In such a case, command authority must not be compromised by software processing demands.

The code is specifically designed to avoid considerable delay in processing the thruster command, even when the server GUI is currently carrying out a processing-heavy task. This responsiveness was achieved through the use of multi-threading [11], which allows the code to execute different tasks on different threads to model simultaneity. The server code is specifically designed to relegate each server task to its own processing "thread" to prevent any tasks from being stalled while the server is managing a different task. In this manner the tasks associated with requesting, receiving, processing, displaying, and relaying the telemetry data, as well as receiving and issuing thruster commands, were assigned to different threads to allow the server GUI to manage several tasks nearly simultaneously without loss of responsiveness. This responsiveness is a drastic departure from previously used test code, which was 
designed to either check telemetry data or send commands to the jetpack. Although the avionics box can only manage a telemetry status check or thruster command, the server GUI can do both nearly concurrently, so that the server can request telemetry data from the avionics box and then send it out to the headset without impeding the operator's ability to send thruster commands.

\section{Controller Hardware}

The legacy SAFER avionics unit has two main control ports. One is for connecting the HCM and the second is available for ground testing. This port uses as standard DB9 serial connector that interfaces to a laptop with customized software that runs a series of start-up tests to check for system readiness. The ground testing port is used to interface the two hands-free control methods via the server laptop. The following section describes the evolution of the hardware testing in parallel with the software maturation.

Various hardware devices were selected to test and implement the hands-free voice and foot sensor control modes. Early voice control testing (shown in Figure 1) utilized "theBoom" noise-cancelling microphone to achieve hands-free control and verify the implementation and control software. Implementing this microphone entailed use of the voice recognition engine in windows to identify the voice commands using $\mathrm{C} \#$ class libraries. The taxonomy created recognizes a specific phrase (as described in section 2) and triggers an appropriate custom function specified in the software. These words were then translated to the Hexadecimal equivalent string command that would be sent through the serial port to the avionics box. The duration of the thrust-open was fixed at 4 pulses of 1 second each, though this can be easily changed in the code as desired.

After initial success with the microphone and demonstrating the ability to translate verbal speech into thruster commands, headset and foot sensor testing began. The headset processor runs a Windows Mobile OS in the background with customized software. As verbal commands are issued to the jetpack and the telemetry received, it is displayed on the computer screen eyepiece. Though a USB interface is available, it would restrict the operator's movements; therefore $\mathrm{Wi}-\mathrm{Fi}$ is primarily used to communicate with the server laptop

To implement the foot sensor control method, three Tekscan pressure sensors are mounted on an adjustable plastic base that in turn is mounted onto adjustable aluminum railings, one near each of the operator's feet (see Figure 9). Each sensor was mounted individually on a thin plastic backing that was slotted to allow for adjustability against a slotted mounting base to accommodate the NASA anthropomorphic database standards of $5^{\text {th }}$ percentile female to $95^{\text {th }}$ percentile male foot geometry. Described further in section 6 , these plates were designed to provide a "resting" location for the operator's foot. As this testing is done in a $1-\mathrm{g}$ environment, the free-floating boot arrangement is replicated by requiring the operators to be seated on the test stand. The operators can then off-load their weight and lightly place their foot on the plate and press down with the appropriate segments of the foot for control. Operators are in stocking-feet during foot sensor control to more accurately reflect the in-flight method.
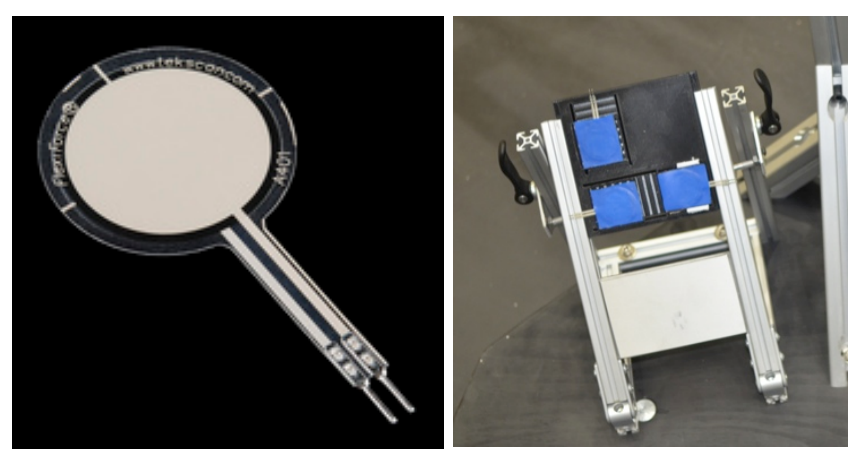

Figure 9 - Left: Close up of the force sensor and Right: the three sensors mounted under the big toe, ball of foot and outstep, on an adjustable plate

The pressure sensor consists of a piezoresistive force sensor that varies its resistance in response to pressure applied to it. If no pressure is applied, the sensor will register $20 \mathrm{Mega}$ ohms or higher, up to infinite resistance. As pressure is applied the resistance reduces in proportion down to zero ohms. When one of the sensor resistances crosses a predetermined threshold it will trigger a function in the Netduino to communicate to the server laptop, which will in turn send the key word for the corresponding thrust command to the avionics unit. A potentiometer is used to adjust the threshold level for all the sensors and can be adjusted real time depending on the operator's resting force profile.

The sensors are connected to a "Netduino Plus" electronics board with the .NET micro framework, which is used to communicate with the server laptop. The Netduino Plus was chosen for its compatibility with the .NET Micro Framework used in $\mathrm{CH}$ and because it allows for re-use of the code. Although Netduino is a hobby grade microprocessor, it is a good starting point for the proof of concept testing, offers a good introductory platform for rapid design, and can be easily upgraded to other processors with more capabilities in the future if needed.

\section{TEST BED HARDWARE}

The engineering test bed hardware, shown in Figure 10, was designed and constructed to provide a suitable air bearing method to test the new jetpack by compensating for the residual friction and gravity of the test environment. This section details the test bed hardware, namely the mechanical components necessary to operate the jetpack using the ABF, which is an extremely smooth, flat surface that allows articles to float on air bearings thereby simulating near frictionless motion. The mechanical components include an air-bearing sled to support an operator and jetpack while gliding on the $\mathrm{ABF}$ under jetpack thruster power, and a 
mobile tower to ferry the shop air supply to the air bearings on the sled. These two components provide the means for the jetpack to be thoroughly tested on the ABF to validate its operational performance while also analyzing the effectiveness of the two different hands-free control methods.

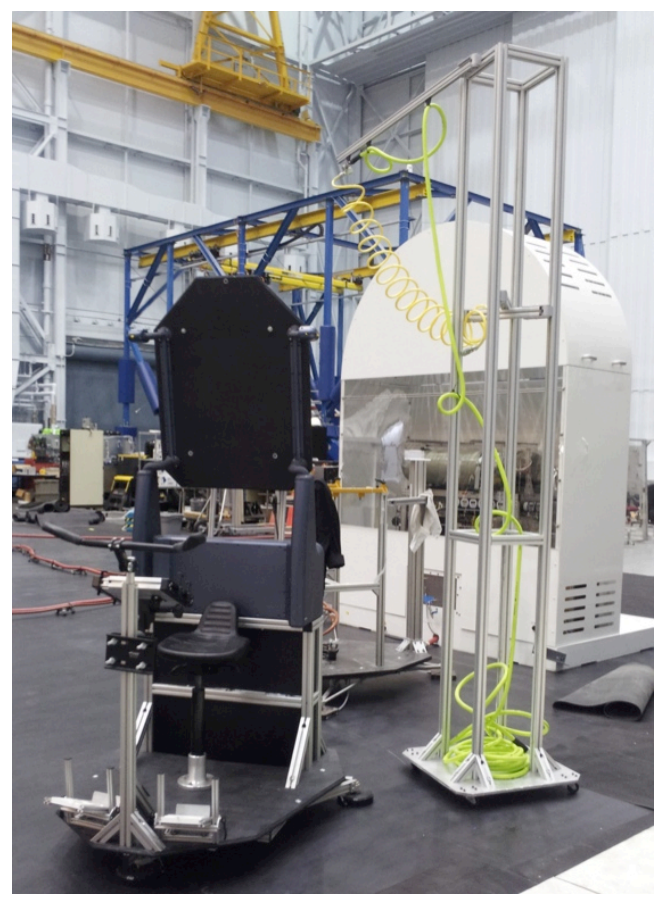

Figure 10 - Left: Engineering test unit air sled and jetpack hardware

\section{Air Sled Hardware and Considerations}

The weight of the air sled is supported by three circular air bearings that are mounted under a triangular aluminum frame to allow the entire assembly to smoothly glide on the ABF. The air bearings are supplied via a nylon hose plumbing system that transports the shop air from the attachment point at the top of the sled to each air bearing. A sheet of plywood is mounted on top of the aluminum frame for the operator to stand on. Additionally, a separate aluminum frame mounts the jetpack to the plywood base while supporting it at an appropriate height off the platform to mirror the placement of the jetpack when integrated with an EVA suit. The aluminum frame that connects the jetpack to the plywood base also functions as a housing unit for the avionics box, power supply, and server laptop. Though the jetpack design was meant to demonstrate hands-free capabilities, a handlebar is mounted at the front of the air sled to give the operator additional stability, particularly when mounting and dismounting the test stand. A modified sit-stand stool is mounted just in front of the jetpack to provide the operator with a method to offload his/her weight while operating the foot sensors, as described previously. The foot sensors were placed on custom-designed variable incline plates, which were bolted to the plywood base on both sides of the handle bar mount near the operator's feet. Lastly, to further accommodate the variation in anthropometric sizing, the human interface components of the test stand are all adjustable. The seat is mounted on a hydraulic pole for vertical travel and can also tilt and rotate. The foot sensors are mounted onto a custom designed aluminum frame that can adjust its incline with quickrelease handles. The handlebars are mounted using an adjustable stem that can both rotate and slide up and down on a vertical aluminum pole to allow for change in incline and vertical travel.

There were many factors to be considered and balanced throughout the development of the test hardware. First and foremost, the magnitude and distribution of the air sled's weight was an extremely important factor in the design process. Minimizing the weight of the entire air sled was critical to maximizing the sled's performance on the ABF, in that as the weight of the sled grew, it became less responsive and more difficult to control. In designing the lower aluminum frame, plywood base, and aluminum frame supporting the jetpack, each component was minimized to drastically cut back on weight. Beyond simply minimizing the weight of the system, the location of the center of gravity (CG) was tightly controlled to maintain an even loading on each air bearing. This was desirable because an even loading on each air bearing results in a symmetric frictional drag force about the $\mathrm{CG}$ from all three air bearings during motion. In other words, if the air bearings each supported a significantly different load they would induce a moment about the CG whenever the air sled was in motion. Though this effect would be nullified by the jetpack's control system in the avionics software, it would reduce efficiency by wasting propellant and was therefore undesirable. The air sled was therefore designed such that the CG stayed within a "safe zone" (illustrated in Figure 11) where the load on each air bearing was within $5 \%$ of the average. To accurately fulfill the weight and CG requirements of the design, the mass properties of every new component and several existing components had to be assigned or estimated in the CAD model using material data. Once the material properties of every component in the model were defined, the CAD software was used to calculate the location of the CG of the entire assembly.

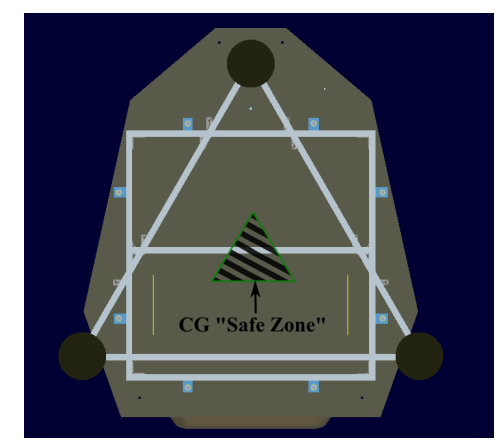

Figure 11 - Graphical of the test stand base showing the location of the air bearings, CG and safe zone

The plumbing that connects the shop air supply to the three air bearings was also designed with a number of factors in 
mind. Nylon tubing was chosen for its flexibility and excellent compatibility with push-to-connect fittings to create a quick airtight seal while also maintaining the possibility of a quick disconnect for reconfiguration. The inner diameter of each plumbing component was maximized within reasonable limits to minimize pressure loss along the plumbing system. Additionally, industrial socket connections were placed between the shop air supply and hose tower, as well as between the hose tower and air sled, allowing easy disconnect of any component in the air supply chain.

After designing the aluminum frame that mounts the jetpack to the plywood base, it became apparent that the jetpack's upper plate had a long moment arm on its attachment point and would most likely oscillate in the pitch direction during operation in a $1 \mathrm{G}$ environment (illustrated in Figure 12). Because this issue will only be problematic in a $1 \mathrm{~g}$ environment, the solution is an external structure not associated with the jetpack, rather than a modification of the jetpack design. To counter the possible pitching instability, a rear support structure was designed to run up the back of the jetpack and firmly attach the upper jetpack plate to the aluminum support structure below. The rear support structure was also designed to hide inside the PLSS mockup attached to the rear jetpack plate for aesthetic purposes.

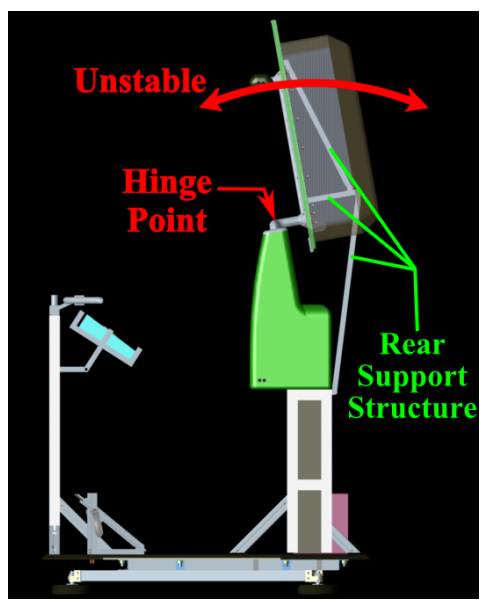

Figure 12 - Graphical model of the test stand showing the rear support structure needed for overall stability

\section{Mobile Hose Tower Hardware and Considerations}

To ferry the shop air to the air bearings on the sled a mobile tower, shown in Figure 8, was designed and built with the intention of minimizing the drag experienced by the airbearing sled due to the air hose (as was known to have plagued the previous SAFER ABF test stand). Recall that the air bearings on the sled are powered by the shop air supply, whereas tanks housed inside the unit supply the thrusters on the jetpack. The tower is intended to loosely follow the air sled around the ABF while being lightly pushed by test support personnel. To avoid tangling the shop air hose with the air sled, the mobile hose tower stands over ten feet tall and the tower itself has a 10"x10" cross section. A four-foot arm extends out from the top of the tower and carries the air hose out to the air sled.

The ten foot tall structure could have easily had a possible inherent tipping instability due to its relatively tall height compared to its base area. To avoid this, two solutions were implemented. First, the aluminum base to which the tower mounts is a $3 / 4$ " thick aluminum plate with a square area two feet by two feet. Second, low-clearance casters were mounted to the underside of the aluminum plate to enable tower mobility. These two solutions keep the CG close to the ground and provide a wide support base for the entire tower. After assembly, it was clear that these two solutions were more than adequate to maintain the tower's stability.

The mobile hose tower has a number of devices intended to drastically reduce the drag induced by the shop air hose on the air sled. First, it has casters on the bottom that allow it to roll around the floor and loosely follow the air sled. At the top of the tower, the arm extends out away from the tower and can swing back and forth on a hinge to track the sled. The hose follows the arm out away from the tower by attaching to three sliders that can move back and forth along the length of the arm. At the end of the arm, a 2 DOF rotating joint allows a coiled hose to point in any direction towards the sled. The coiled hose then travels to the air sled, where it connects to another rotary joint that further increases the flexibility of the system.

\section{Testing}

The hardware and software system was tested in several environments that provided a continual and iterative testing process as the jetpack was being constructed in parallel. As described previously, software was tested initially by attaching the server laptop to the existing SAFER system to verify the end-to-end (voice-to-thruster or foot-to-thruster) code function. Additional testing and verification was performed in the NASA Johnson Space Center Virtual Reality (VR) lab, where astronauts train to fly SAFER on board the International Space Station (ISS). Using a fullfidelity simulation capability, a virtual 3D image of the ISS and virtual astronaut equipped with the SAFER jetpack are displayed to the user through a head mounted display. Astronauts use a physical hand controller to command SAFER in the virtual world; however for testing purposes, a communication port cable was used to bypass the hand controller such that the server laptop could be connected. Not only is the VR a useful facility for checkout, but it will provide a training capability to future operators of the jetpack, similar to current training techniques. After completing an end-to-end system test in the VR lab, a final test was performed with the actual SAFER avionics and new jetpack test stand. This confirmed the successful operation of the GUI code and validated the software and hardware to run the hands-free control systems.

Lastly, once the jetpack thruster hardware was delivered and it's operation verified, an fully-integrated ABF checkout of both hands-free methods was possible. Both the voice and 
foot sensor checkouts were successful. However, after several minutes of testing the headset application became unstable and stopped working due to the extremely high levels of Wi-Fi interference from competing systems in the hi-bay building that would drop the connection to the headset, causing the GUI to quit. In addition to finding ways to manage and affect the number of wireless devices in the hi-bay, the next software revision will be made more robust to dropped connections and interference. Previous testing up to this point had not surfaced this bug, since this was the first full facility activity environment test. If $\mathrm{Wi}-\mathrm{Fi}$ interference cannot be affected, the software must be augmented to continually monitor and status this connection as well as reconnect automatically in case of dropped connections. The foot pedal however, was very reliable and thruster firing executed correctly when any of the sensors were pressed. The foot pedal did not suffer from Wi-Fi interference since it is connected via Ethernet to the server controller.

Despite the lack of drag from the mobile tower, the sled motion across the air-bearing floor still required two or three thruster commands in the same direction to start significant movement, overcome any residual friction and significantly propel the fully loaded sled. From a human factors perspective, it was noted that when mounting the sled, the operator needed to ensure that they did not carry any residual momentum in any direction or the sled would act as a "skateboard" once the subject was onboard. The sled is also sensitive to torso movement that causes a rotation similar to a yaw thrust that needs to be nulled out. It was also noticed that the operator needs to be seated upright and not lean in any direction or the center of gravity would shift enough that a command such as $+/-Y$ created a minor unintended "yaw" if the subject was leaning forward.

\section{Conclusions and Future Testing}

This paper has detailed the design, development and implementation of a hands-free control system and test capability for a new EVA jetpack. As can be seen from early checkout testing, the new control modes can successfully act in place of the hand controller, provide EVA mobility and expand future EVA capabilities. There are some unique adjustments that must be made to the test sled and test environment as the project progresses. Areas of improvement have surfaced for the locations of the foot sensors, updates to the software, and modifications to the test sled itself. As the engineering evaluations continue, possible improvements to consider include upgrading the foot sensor controller to a Field Programmable Gate Array (FPGA), replacing the laptop with a .NET microprocessor or FPGA, and developing customized Printed Circuit Boards (PCB) to reduce the overall footprint of the design.

A microgravity flight is currently being planned to test the helmet and foot sensors in a more high fidelity environment. The foot sensors will be integrated into a EVA-like boot where the true capability and applicability of an in-suit sensor controller can be evaluated. This part of the research would be done in collaboration with the NASA Systems Engineering microgravity university program were college students participate in developing a test article with NASA engineer mentorship, then fly the test article and conduct inflight testing.

Another test environment that will be utilized in the near future is the NASA Active Response Gravity Offload System (ARGOS) facility. ARGOS uses a crane assembly to offset a person's weight to simulate gravity ranging from microgravity to other surface gravities (planetary and asteroid). The intent is to add the hands-free interfaces to the system, perform suited off-loading tests and run subjects through EVA tasks to objectively and subjectively quantify the efficiency, accuracy and usability of the systems. As this facility is also located in the hi-bay, the Wi-Fi issue will have to be addressed during integration.

Future plans also include advancing the sophistication of the human interface. Adding range data to the jetpack and using voice along with updated displays to command heading from an egocentric perspective would reduce the numbers of DOF that the operator has to control and leave only the job of controlling the thruster pulse rate. Alternatively, automation can be designed to allow the astronaut to give a meta command (or waypoint) such as "Suitport" and the system would locate the heading and distance to the suitport, and control the orientation and rate of travel, to arrive at a waypoint with close to zero velocity. Trades would need to be analyzed that weighed the increased level of automation with decreased situation awareness and direct control from the astronaut perspective.

\section{REFERENCES}

[1] A. Millbrooke (1998). More Favored than the Birds: The Manned Maneuvering Unit in Space. In P. E. Mack (Ed.), From Engineering Science to Big Science. Washington DC: NASA

[2] “Air Bearing Basics," Nelson Air Corp, New

Hampshire, 2012. Retrieved from

[http://www.nelsonair.com/NA_primer.htm. Accessed 7/27/2012.]

[3] Office of Safety and Mission Assurance, "Formal Methods Specification and Analysis Guidebook for the Verification of Software and Computer Systems", NASA, Washington DC, NASA-GB-O01-97, 1997.

[4] M. Yeh, C. Wickens, F.J. Seagull. "Target Cuing in Visual Search: The Effects of Conformality and Display Location on the Allocation of Visual Attention". Human Factors: The Journal of the Human Factors and Ergonomics Society, 41, 524, 1999.

[5] R. Patterson,M.D. Winterbottom, B.J. Pierce. "Perceptual Cues in the Use of Head-Mounted Visual Displays". Human Factors: The Journal of the Human Factors and Ergonomics Society, 48, 555, 2006. 
[6] A. Jaimes, N. Sebe. "Multimodal Human Computer Interaction: A Survey" IEEE International Workshop on Human Computer, 2005.

[7] A. Junker, T. Sudkamp, T. Eachus, et al. Hands-Free Computer Access for the Severely Disabled" National Institute of Child Health and Human Development, 1R43 HD 309070-01.

[8] G.L. Calhoun, G.R. McMillan. "Hands-Free Input Devices for Wearable Computers". Proceedings of the Fourth Symposium on Human Interaction with Complex Systems, 118, 1998.

[9] C. Parkinson. "Golden-I Headset Computer," Kopin Corportation, Maryland 2011. [http://www.mygoldeni.com/. Accessed 7/27/2012.]

[10] M.A. Thornton, "A Survey and Engineering Design of Atmospheric Diving Suits," Master of Engineering thesis, Ocean Engineering, Texas A\&M University, 2000.

[11] Asche, R. Ruediger. "Multithreading for Rookies" Microsoft Developer Network, created 1993. [http://msdn.microsoft.com/en-us/library/ms810438.aspx. Accessed 10/15/2012]

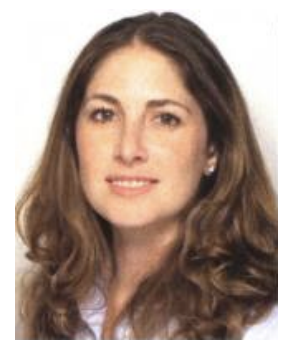

\section{BIOGRAPHIES}

Jennifer Rochlis Zumbado received a B.A. in Physics from Mount Holyoke College and an M.S. and Ph.D. in Aeronautics \& Astronautics from MIT in 2001. She has been at the NASA Johnson Space center for 12 years. A human factors engineer, human systems integration lead and human-robotic systems designer, she has worked on projects such as Robonaut, the next generation Lunar/Martian rovers, ground control of Space Station and Space Shuttle arms, and is the Principal Investigator for the Human Research Program Human-Robotic Interface Project.

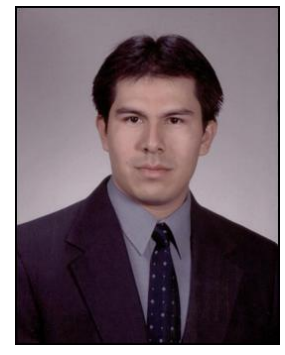

Pedro H. Curiel received a B.S. in Electrical Engineering from Instituto Tecnologico Cd Juarez, a MS of Industrial Engineering as well as MA Business Administration from New Mexico State University in 2003. He started at NASA Stennis Space Center and now has been at NASA Johnson Space Center for 7 years. He began as a Risk Manager for Stennis, then later as a systems engineer on the Constellation program. He is now working in the Software and Robotics branch on the Hands Free Jetpack and Modular Robotic Vehicle projects.

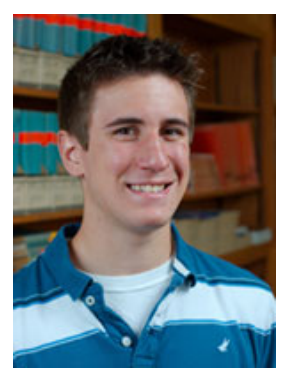

Samuel S. Schreiner is currently pursuing a Bachelors of Aerospace Engineering at the Universtiy of Minnesota where he is also pursing a Management Minor and studying Chinese. He contributed to the EVA Jetpack project during his internship in the summer of 2012 at the Johnson Space Center. At the University of Minnesota, he worked for 3 years as a research assistant in the Physics department where he contributed to two publications and was the primary author for a third. Currently, he is carrying out a research project with the Aerospace Department studying the performance of a unique airfoil design. 
